\title{
POSSIBILITIES OF INDIVIDUAL CLAIM RESERVE RISK MODELING
}

\author{
Pavel Zimmermann*
}

\section{Introduction}

A significant increase in demand for insurance and financial risk quantification has occurred recently due to the fact that the implementation of a complex of rules of international reporting standards (IFRS) and solvency reporting (Solvency II) has started. It appears that the key question for solvency measuring is the estimation of the probability distribution of future cash flows of an insurance company. That means it is required to model 'how much' and 'when' the company is liable to the insured. Solvency is then reported through an appropriate risk measure based, e.g., on a percentile of this distribution.

While the present popular models (see, e.g., Mack, 1993; England and Verrall, 2002; England and Verrall, 2006; or (Merz and Wuthrich, 2008) are based solely on aggregated data (such as total loss development for a certain time period), it is certainly challenging from both the theoretical and the practical point of view to scrutinize possibilities of modeling the reserve risk (i.e., roughly said, the distribution of the ultimate incurred value of claims that have already happened in the past) based directly on individual claims. Although it is obvious that this kind of modeling is necessary to properly include some individual properties such as limits or the re-insurer's share, this kind of models has not yet become popular.

Several such approaches have already been published. One can distinguish articles focused on theoretical aspects of the underlying processes and ones focused on the practical aspects. An interesting inconsistency appears between these two groups of models. While the theoretically oriented models (such as Norberg, 1993 or Arjas, 1989) stress the continuous time aspect of the individual claim random processes approach, models that are oriented more on the practical side of the problem (mostly those published on the grounds of consultancy companies, such as Taylor et al., 2008 or Murphy and McLennan, 2006) construct their models based on discrete time points (usually consecutive development years). In this paper, an attempt to overcome this paradox is made. The development of an individual claim (the settlement process) is not modeled in consecutive development years. Instead, the so-called stages of the settlement process are introduced and defined as the time periods between changes

* University of Economics, Prague, Faculty of Informatics and Statistics (pavel.zimmermann@vse.cz).

This paper was supported by internal grant of University of Economics, Prague, number F4/25/2011. 
in the incurred value. It is shown that under practically acceptable assumptions, the handling time model (model of the time from occurrence to settlement) is separated from the model of the ultimate incurred value (final loss) of the claim.

It is apparent that the key element of such models is the distribution of the future liability that stems from an individual claim. In this paper, we will compile the theoretical background of the modeled random processes and then we will focus on possibilities of practical modeling of an individual claim. Thus, we will focus on:

1. Assumptions that would significantly simplify the model on the one hand and could still be considered 'acceptable' from the practical point of view on the other hand;

2. Decomposition of the problem into 'sub-problems' that can be solved under real conditions.

The main focus will be on a model of the settlement process of an individual claim. Aggregation of the risks will not be within the scope of this paper. It is, however, anticipated that Monte Carlo simulations will be used for this purpose.

In Section 2, some basic terms of the settlement process are introduced and the process is described formally. Main variables and their notation are introduced here. In Section 3, the basic structure of the model is outlined and simplifications that allow separating the component of the incurred value changes (i.e., risk of the reserve revision) and the timing component are set. In Section 4, the model of the incurred value changes is derived. Some further simplifying assumptions are made and commented on from the practical point of view. One of the main results of this article, the probability density function of the ultimate incurred value (i.e., the ultimate loss) is derived at the end of this section. The other component of the model, i.e., the distribution of the time to settlement, is considered in Section 5. The decomposition of the problem into the above mentioned components allows using standard statistical procedures for estimating them. One of the components, however, seems to be particularly challenging: the model of the size of the incurred value changes. In Section 6, a joint modeling procedure is applied to this particular component and some interesting practical properties are demonstrated on real data.

\section{The Ultimate Incurred Value}

\subsection{Practical Description}

First, we formulate the problem from the practical point of view and we briefly introduce some basic terms. The insurance company has to set up reserves (technical provisions) until all claims are paid out to preserve its solvency. We can distinguish three main types of reserves:

1. The reserves on claims that occurred, were reported but are not yet settled.

2. The reserves on claims that occurred but were 'not enough' reported.

3. The reserves on claims that occurred but have not yet been reported.

The first type of reserves is usually the responsibility of the claim handling department. This type of reserves is always assigned to a specific claim. These reserves 
are usually called the 'case reserves' or the 'RBNS reserves' (reported but not settled). When a claim occurs and is reported to the insurance company, the claim handler collects all available information and sets up this reserve. This reserve basically reflects the claim handler's opinion on the unknown random ultimate loss paid to the insured. As more and more information about the claim becomes available the claim handler updates his estimate and changes the reserve. When all the necessary information is collected (or the trial is ended, etc.), the claim is settled and paid out. This process will be referred to as the settlement process. Sometimes the claim may be partially paid out before the settlement. Then, the corresponding amount of the reserve is released. For this reason, another term is commonly used: the so-called 'incurred value'. The incurred value of a claim is the amount already paid out plus the appropriate reserve assigned to this claim. That means that the incurred value is a general term that represents the presently estimated value of the claim (no matter whether the claim is paid out or not). The potential unfavorable evolution of the incurred value needs to be covered from the reserves on claims that are incurred but not enough reported (IBNER).

The third type of reserves, i.e., the reserves on claims that incurred but have not yet been reported (also called the 'IBNR reserves') is the responsibility of the actuaries. Based on the historical observations and statistical methods, the total amount of claims that are yet to be reported is estimated. This type of reserves is not assigned to individual claims but rather to some aggregate.

From a risk management point of view, it is needed to determine the risk that all these reserves will not be sufficient to cover future liabilities. That means that an additional 'second level' estimate is commonly produced. This estimate concentrates on the estimate of volatility and perhaps also on the potential bias of the 'first level' estimates (i.e., measures whether the previously mentioned claim handlers' and actuaries' estimates are not systematically under- or overstated and what is their accuracy).

\subsection{Formal Description of the Settlement Process}

Papers on this topic (such as Arjas, 1989 or Norberg, 1993) generally use marked processes for the description of the settlement process. We will adopt a similar description of the problem in this section. We assume that for a claim that occurred at a certain time point $T$, there are two marked processes:

1. $\left\{\left(X_{s}, T_{s}\right) ; s \geq 1\right\}$, where $X_{s}$ are changes in the incurred value (the adjustments) of the claim and $T_{s}$ are the time points of the changes in the incurred value for which

$$
T<T_{1}<T_{2}<T_{3} \ldots
$$

2. The delay $D \equiv T_{1}-T$ is then the reporting delay. The period between two consecutive events will be referred to as the stage of the process in this paper. (The term 'stage' is not an official term.)

3. $\left\{\left(P_{s^{\prime}}, T^{\prime}{ }_{s^{\prime}}\right) ; s^{\prime} \geq 1\right\}$, where $P_{s^{\prime}}$ is the payments corresponding to that claim, and $T^{\prime}{ }^{\prime}$ is the corresponding time points for which 


$$
T<T_{1}<T^{\prime}{ }_{1}<T^{\prime}{ }_{2}<T^{\prime}{ }_{3} \cdots
$$

4. Notice that $T_{1}<T^{\prime}{ }_{1}$ due to the fact that some amount of reserve has to be set up prior to the first payment.

These processes are inter-related in many aspects. Some basic relations hold between the cumulative processes defined as:

and

$$
Y(t) \equiv \sum_{s: T_{s} \leq t} X_{s}
$$

$$
Q(t) \equiv \sum_{s^{\prime}: T^{\prime} s^{\prime} \leq t} P_{s^{\prime}} .
$$

One practical property is that the inequality

$$
Q(t) \leq Y(t)
$$

holds for each $t$. Another useful relation is that after a sufficient amount of time, the whole claim will be paid out and the two cumulative processes will be equal, i.e.,

$$
Q(\infty)=Y(\infty)
$$

The variable $Y(\infty)$ is called the ultimate incurred value; it represents the final loss generated by the claim and therefore is the most important variable to be modeled. For the sake of simplicity, this variable will be denoted simply as $Y$. In this article, we will consider the so-called stages of the settlement process. The $s$-th stage will be defined as the time period between changes in the incurred value, i.e., the interval $T_{s}<t<T_{s+1}$. During the $S$-th stage, the incurred value remains constant and will be denoted as $Y_{s}$, i.e.,

$$
Y_{s} \equiv Y(t) \quad \text { for } \quad T_{s} \leq t<T_{s+1} .
$$

\subsection{Closed Claims}

Practically, there is only a finite number of nonzero adjustments $\left\{X_{s}\right\}$ or payments $\left\{P_{s^{\prime}}\right\}$ respectively. The number of the nonzero adjustments or payments will be denoted as $S$ or $S^{\prime}$ respectively. The claim is usually referred to as 'closed' or 'settled' after $T_{S^{\prime}}$. Formally, we can consider (as in Arjas, 1989) that after the settlement, all changes in the incurred value or payments equal to zero and their time points are equal to infinity.

In this article, we add extra marks to the processes. Namely, we add a dummy variable ('indicator') $A_{s}$ (or $A^{\prime}{ }^{\prime}$ ' respectively) representing the open/closed status of the claim, i.e., defined as 


$$
\begin{aligned}
& A_{s}=1 \quad \text { for } \quad s: T_{s+1}<T_{S^{\prime}} \\
& A_{s}=0 \quad \text { for } \quad s: T_{s+1} \geq T_{S^{\prime}}
\end{aligned}
$$

that is, $A_{s}=1$ for stages in which the claim is open and $A_{s}=0$ for stages in which the claim is closed. $A_{s^{\prime}}^{\prime}$ can be defined analogously.

\section{The Model Structure}

\subsection{The Task}

The time point up to which the information about the history is available will be referred to as the present time and will be denoted as $\tau$. At this time, the main task of the modeler is to estimate the amount yet to be paid out and the timing of the future payments. The latter problem needs to be answered due to the fact that the time value of money should be considered. (Determination of the investment strategy is not within the scope of this paper.) This means we need to model the conditional distribution of a random variable which is some transformation (e.g., present value) of the random vector $\left(T^{\prime}{ }_{1}, T^{\prime}{ }_{2}, \ldots, T^{\prime}{ }_{S^{\prime}}, P_{1}, P_{2}, \ldots, P_{S^{\prime}}\right)$ conditioning on the history of the claim known up to the modeling time $\tau$. This history will be denoted as $H_{\tau}$.

\subsection{Payments or Incurred Value}

In the above paragraph, the incurred value is not mentioned explicitly as the required output of the model. However, in portfolios with longer development, it is beneficial to base the estimate of the ultimate incurred value on the development of the incurred value $Y(t)$, which typically converges to the ultimate incurred value significantly faster than the development of the payments $Q(t)$. Thus, it is assumed that there is more information about the ultimate incurred (or, equivalently, ultimate paid) value contained in the incurred value history than in the paid amount history. This approach, however, still requires the estimate of the payments as well as their timings in order to be able to include the time value of money (discounting). That means that the vector $\left(T^{\prime}{ }_{1}, T^{\prime}{ }_{2}, \ldots, T^{\prime}{ }^{\prime}, P_{1}, P_{2}, \ldots, P_{S^{\prime}}, Y\right)$ would need to be estimated (conditioning on $H_{\tau}$ ).

\subsection{Potential Simplifications}

It is quite obvious that a model with so many mutually dependent variables would be very difficult to construct. Therefore, suitable simplifications should be searched for to simplify the model as much as possible while keeping the assumptions more or less realistic. Since under 'normal' economic conditions it can be expected that discounting is connected with lower risk than the revision of the claim value, it seems reasonable to simplify mainly the 'time variables'. The task can be greatly simplified if we can assume that the ultimate incurred value of an individual claim is paid all at once at some time $T^{*}$. This reduces the task to modeling only the random vector $\left(T^{*}, Y\right)$ (conditioning on $H_{\tau}$ ). This simplification further allows decomposing the problem into a reserve revision risk problem (i.e., the change in the claim handlers' estimate of the incurred value) and the timing problem. It is possible to take advantage of the relation 


$$
f_{T^{*}, Y}\left(t^{*}, y \mid H_{\tau}\right)=f_{Y}\left(y \mid H_{\tau}\right) f_{T^{*}}\left(t^{*} \mid y, H_{\tau}\right)
$$

where $f$ denotes the corresponding probability distribution function. This immediately suggests modeling the two components sequentially.

\section{Reserve Revision Risk}

In this section, we will consider the first multiple of the right-hand side of equation (9), i.e., $f_{Y}\left(y \mid H_{\tau}\right)$. The history $H_{\tau}$ consists of the knowledge of values of several random variables, namely the knowledge of:

1. the (changes in the) incurred value $\left\{X_{s}, s: T_{s}<\tau\right\}$ of all claims in previous stages;

2. the times of the changes in the incurred value $\left\{T_{s}, s: T_{s}<\tau\right\}$ of all claims in the previous stages;

3. the previous payments $\left\{P_{s^{\prime}}, s^{\prime}: T^{\prime}{ }_{s^{\prime}}<\tau\right\}$ of all claims;

4. the times of the previous payments $\left\{T^{\prime}{ }_{s^{\prime}}, s^{\prime}: T^{\prime}{ }_{s^{\prime}}<\tau\right\}$;

5. potential 'external' information.

To determine the distribution of the ultimate incurred value, we need to choose the information that is relevant on the one hand and still manageable in terms of modeling on the other hand. At the time of modeling (the time $\tau$ ), every claim is in a different stage and has a different history. Therefore, every claim will have a different conditional distribution of the ultimate incurred value. The elementary property is that once the claim is closed, there is no randomness or risk in the claim and the ultimate incurred value equals the present (i.e., at $\tau$ ) incurred value (or, equivalently, paid value). Augmenting the model for re-opening of claims is technically possible but this feature would require significantly more operations and the materiality of re-openings is often negligible, therefore re-openings are not considered in this paper. We denote the present stage of the claim as $S_{\tau}$. We can write for the conditional distribution of the ultimate incurred value of the presently open claim:

$$
f_{Y}\left(y \mid H_{\tau}\right)=\sum_{s=s_{\tau}}^{\infty} f_{Y_{s}, s}\left(y, s \mid H_{\tau}\right) .
$$

(Notice that the value of $Y_{s_{\tau}}$ is known at the time $\tau$. Therefore, the distribution $f_{Y_{s}, S}$ is degenerated for $S=S_{\tau}$.)

This probability density can be further rewritten considering the following relationship

$$
f_{Y_{s}, s}\left(y, s \mid H_{\tau}\right)=f_{Y_{s}}\left(y \mid H_{\tau}\right) f_{s}\left(s \mid Y_{s}=y, H_{\tau}\right) .
$$

This approach seems to be beneficial for modeling because (analogously to 'traditional' aggregate models) it allows modeling of the claim development in each future stage. (Notice, however, that the aggregate models consider discrete time intervals 
instead of stages.) It is possible to consider the distribution of the incurred value as an integral 'over all trajectories', i.e.,

$$
\begin{aligned}
& f_{Y_{s}}\left(y \mid H_{\tau}\right) f_{S}\left(s \mid Y_{s}=y, H_{\tau}\right)= \\
& \int_{\Omega_{s_{\tau}}} \int_{\Omega_{s_{\tau}+1}} \ldots \int_{\Omega_{s-1}} f_{Y_{s_{\tau}}, Y_{s_{\tau+1}}, ., Y_{s}}\left(y_{s_{\tau}}, y_{s_{\tau}+1}, \ldots, y \mid H_{\tau}\right) . \\
& f_{S}\left(s \mid y_{s_{\tau}}, y_{s_{\tau}+1}, \ldots, y, H_{\tau}\right) d y_{s-1} d y_{s-2} \ldots d y_{s_{\tau}} .
\end{aligned}
$$

Since the event that the claim will be closed in the $S$-th stage, i.e., the event $S=S$ is equivalent to the event the claim will remain open until $S$ and will be closed in $S$, that is $\left(A_{1}=1, A_{2}=1, \ldots, A_{s-1}=1, A_{s}=0\right)$. (Remember that no reopening was considered here, therefore this event already implies that $A_{s+1}=0, A_{s+2}=0, \ldots$ ) This means that we can write further

$$
\begin{aligned}
& f_{Y_{s}}\left(y \mid H_{\tau}\right) \cdot f_{S}\left(s \mid Y_{s}=y_{s}, H_{\tau}\right)= \\
& \int_{\Omega_{s_{\tau}}} \int_{\Omega_{s_{\tau}+1}} \ldots \int_{\Omega_{s-1}} f_{Y_{s_{\tau}}, Y_{s_{\tau+1}}, ., Y_{s}}\left(y_{s_{\tau}}, y_{s_{\tau}+1}, \ldots, y \mid H_{\tau}\right) . \\
& \cdot f_{\mathrm{A}_{s_{\tau}}, A_{s_{\tau}+1}, ., A_{s}}\left(1,1, \ldots, 0 \mid y_{s_{\tau}}, y_{s_{\tau}+1}, \ldots, y, H_{\tau}\right) d y_{s-1} d y_{s-2} \ldots d y_{s_{\tau}} .
\end{aligned}
$$

This means that we can further separate the problem into a model of the incurred value development given that the claim is open and the probability that the claim remains open until $S$, when it is closed. At this stage, some further simplifications will have to be employed. In similar cases, some independence assumptions are used for the vector $\left(Y_{1}, Y_{2}, \ldots, Y_{s} \mid H_{\tau}\right)$. The most common is probably the Markovian property. Of course the validity of this assumption is not guaranteed for all portfolios. In general it seems to be a reasonable approximation of reality which simplifies the model significantly. If we accept this assumption, we can write

$$
\begin{aligned}
& f_{Y_{s_{\tau}}, Y_{s_{\tau+1}}, ., Y_{s}}\left(y_{s_{\tau}}, y_{s_{\tau}+1}, \ldots, y_{s} \mid H_{\tau}\right)=f_{Y_{s_{\tau}}}\left(y_{s_{\tau}} \mid H_{\tau}\right) f_{Y_{s_{\tau}+1}}\left(y_{s_{\tau+1}} \mid y_{s_{\tau}}, H_{\tau}\right) . \\
& \cdot f_{Y_{s_{\tau}+2}}\left(y_{s_{\tau}+2} \mid y_{s_{\tau}+1}, H_{\tau}\right) \cdot \ldots \\
& \ldots f_{Y_{s}}\left(y_{s} \mid y_{s-1}, H_{\tau}\right) .
\end{aligned}
$$

This form finally allows for fitting the distributions on the data using a more or less 'standard' statistical procedure. Of course, the simplest choice in this case is to assume that only the previous stage incurred value is relevant for the modeling. More complex assumptions are also possible if felt necessary. Some variables contained in $H_{\tau}$ can be considered. Notice the analogy of the right-hand side multiples with the stochastic development factors common in aggregate models. 


$$
f_{A_{s_{\tau}}, A_{s_{\tau}+1}, \ldots, A_{s}}\left(1,1, \ldots, 0 \mid y_{s_{\tau}}, y_{s_{\tau}+1}, \ldots, y_{s}, H_{\tau}\right) .
$$

Now, we will consider the distribution

Again, it seems reasonable to model the probability of the claim remaining open or getting closed 'stage by stage'; therefore, we can write

$$
\begin{aligned}
& f_{A_{s_{\tau}}, A_{s_{\tau}+1}, ., A_{s}}\left(1,1, \ldots, 0 \mid y_{s_{\tau}}, y_{s_{\tau}+1}, \ldots, y_{s}, H_{\tau}\right)= \\
& f_{A_{s_{\tau}}}\left(1 \mid y_{s_{\tau}}, y_{s_{\tau}+1}, \ldots, y_{s}, H_{\tau}\right) . \\
& \cdot f_{A_{s_{\tau}+1}}\left(1 \mid A_{s_{\tau}}=1, y_{s_{\tau}}, y_{s_{\tau}+1}, \ldots, y_{s}, H_{\tau}\right) . \\
& \cdot f_{A_{\tau_{\tau}+2}}\left(1 \mid A_{s_{\tau}}=1, A_{s_{\tau+1}}=1, y_{s_{\tau}}, y_{s_{\tau}+1}, \ldots, y_{s}, H_{\tau}\right) \cdot \ldots \\
& \cdot f_{A_{s}}\left(0 \mid A_{s_{\tau}}=1, A_{s_{\tau+1}}=1, \ldots, A_{s-1}=1, y_{s_{\tau}}, y_{s_{\tau}+1}, \ldots, y_{s}, H_{\tau}\right) .
\end{aligned}
$$

The simplifications to assume here are straightforward. In the simplest case, the independence of the probability of a claim closure on its incurred value can be assumed. This would simplify the calculations to the greatest degree. This independence can, however, only be assumed in some special cases. In general, one should expect that larger claims are more complicated to handle and tend to pass through more stages before closure. In such a case, it is probably reasonable to assume that the probability of a claim closure (given that the claim is open) depends at least on the present incurred value. (Of course, some further variables contained in $H_{\tau}$ might be added if felt necessary.) Thus, we can assume that

$$
\begin{aligned}
& f_{\mathrm{A}_{\tau_{\tau}}, \mathrm{A}_{\tau_{\tau}+1}, \ldots, \mathrm{A}_{s}}\left(1,1, \ldots, 0 \mid y_{s_{\tau}}, y_{s_{\tau}+1}, \ldots, y_{s}, H_{\tau}\right)= \\
& f_{\mathrm{A}_{s_{\tau}}}\left(1 \mid y_{s_{\tau}}\right) \cdot f_{\mathrm{A}_{s_{\tau}+1}}\left(1 \mid A_{s_{\tau}}=1, y_{s_{\tau}+1}\right) \cdot f_{\mathrm{A}_{s_{\tau}+2}}\left(1 \mid A_{s_{\tau}}=1, A_{s_{\tau}+1}=1, y_{s_{\tau}+2}\right) \cdot \ldots \\
& \cdot f_{\mathrm{A}_{\mathrm{s}}}\left(0 \mid A_{s_{\tau}}=1, A_{s_{\tau}+1}=1, \ldots, A_{s-1}=1, y_{s}\right)= \\
& f_{\mathrm{A}_{\tau_{\tau}}}\left(1 \mid y_{s_{\tau}}\right) \cdot f_{\mathrm{A}_{s_{\tau}+1}}\left(1 \mid A_{s_{\tau}}=1, y_{s_{\tau}+1}\right) \cdot f_{\mathrm{A}_{s_{\tau}+2}}\left(1 \mid A_{s_{\tau}+1}=1, y_{s_{\tau}+2}\right) \cdot \ldots \\
& \cdot f_{\mathrm{A}_{s}}\left(0 \mid A_{s-1}=1, y_{s}\right) .
\end{aligned}
$$

We will simplify this further using the notation

$$
f_{A_{j}}\left(1 \mid A_{j-1}=1, y_{j}\right) \equiv z_{j}\left(y_{j}\right) .
$$


Notice also that

$$
f_{A_{j}}\left(0 \mid A_{j-1}=1, y_{j}\right)=1-z_{j}\left(y_{j}\right)
$$

If we now plug equations (13) - (17) back into (10), we get

$$
\begin{aligned}
& f_{Y}\left(y \mid H_{\tau}\right)= \\
& \sum_{s=s_{\tau}}^{\infty} f_{A_{s_{\tau}}}\left(1 \mid y_{s_{\tau}}\right) \int_{\Omega_{\tau+1}} f_{Y_{s_{\tau}+1}}\left(y_{s_{\tau+1}} \mid y_{s_{\tau}}\right) z_{s_{\tau}+1}\left(y_{s_{\tau}+1}\right) \\
& \int_{\Omega_{\tau+2}} f_{Y_{s_{\tau}+2}}\left(y_{s_{\tau+2}} \mid y_{s_{\tau}}\right) z_{s_{\tau}+2}\left(y_{s_{\tau}+2}\right) \ldots \\
& \int_{\Omega_{s-1}} f_{Y_{s-1}}\left(y_{s-1} \mid y_{s-2}\right) z_{s-1}\left(y_{s-1}\right) \\
& f_{Y_{s}}\left(y \mid y_{s-1}\right)\left(1-z_{s}\left(y_{s}\right)\right) d y_{s-1} d y_{s-2} \ldots d y_{s_{\tau}} .
\end{aligned}
$$

Although an analytical solution to equation (19) will not be achievable in the general case, this equation reveals a way for a potential simulation model. The ultimate incurred value of a claim can be simulated by generating the future trajectories of the claim. That is, for each claim and each future stage until closure, we need to generate:

1. Whether the claim was closed or remained open in the given stage (given the incurred value in that stage) from the distribution $z_{j}\left(y_{j}\right)$;

2. Given that the claim was open, the 'jump' in the incurred value given the incurred value in the previous stage from the distribution $f_{Y_{j}}\left(y \mid y_{j-1}\right)$.

Once the claim is closed (and therefore the ultimate incurred value is known), the settlement time $t^{*}$ can be generated given this value.

\section{The Settlement Time}

The previous sections were devoted to the first multiple of the right-hand side of equation (9), i.e., the distribution $f_{Y}\left(y \mid H_{\tau}\right)$. Now we will make some notes on the second multiple, i.e., $f_{T^{*}}\left(t^{*} \mid y, H_{\tau}\right)$. This component represents the distribution of the time to settlement $t^{*}$ given the history and the ultimate incurred value. Of course, the choice of the covariates to condition on will be portfolio dependent. In general, one may expect dependence on the ultimate incurred value $y$ since (as stated above) larger claims are more complicated to settle and tend to be settled later than attritional claims. There is also some information contained in the history $H_{\tau}$. An obvious candidate to include is at least the time already spent in the settlement process. An obvious relation for unsettled claims is then $T^{*}>\tau$. 
As stated in Section 3, it is assumed that the whole claim is paid at once at $T^{*}$ instead of modeling the whole payment pattern

$$
\left(T_{1}^{\prime}, T^{\prime}{ }_{2}, \ldots, T_{S^{\prime}}^{\prime}, P_{1}, P_{2}, \ldots, P_{S^{\prime}}\right)
$$

This means that $T^{*}$ should be some 'sort of average' of the payment times $\left(T^{\prime}{ }_{1}, T^{\prime}{ }_{2}, \ldots, T^{\prime}{ }^{\prime}\right)$. Since the purpose of the inclusion of the settlement time in the model is to include the time value of money, an obvious requirement for this simplification is that the discounted value of the claim (to the time point $\tau$ representing the time of modeling) remains unchanged when using this simplification. Therefore, we require

$$
\frac{\sum_{l=1}^{S^{\prime}} P_{l}}{(1+r)^{\left(T^{*}-\tau\right)}}=\sum_{l=1}^{S^{\prime}} \frac{P_{l}}{(1+r)^{\left(T^{\prime} l^{-\tau}\right)}},
$$

where $r$ denotes the interest rate assumed for discounting. This means we can set

$$
T^{*}-\tau=\frac{\log (Y)-\log \left(Y_{D}\right)}{\log (1+r)},
$$

where

$$
Y_{D}=\sum_{l=1}^{S^{\prime}} \frac{P_{l}}{(1+r)^{\left(T^{\prime} l-\tau\right)}}
$$

is the discounted ultimate incurred value and

$$
Y=\sum_{l=1}^{S^{\prime}} P_{l}
$$

is the (undiscounted) incurred value. This relation can be used to fit the distribution of $T^{*}$ to the closed claims data. In practical tasks, several extensions are needed since one always works with censored data. Some further ideas onthis topic can be found in (Zimmermann, 2010).

\section{Illustration and Properties of the Incurred Value Changes}

In order to be able to use the stochastic model outlined above, one must select appropriate models and data fitting techniques for the components of the model. Whereas calibrating the probabilities of a claim remaining open $z_{j}\left(y_{j}\right)$ and the (conditional) distribution of the time to settlement $f_{T^{*}}\left(t^{*} \mid y, H_{\tau}\right)$ concerns mostly 'common' statistical methods (e.g., logistic regression in the former case and survival analysis in the latter case), setting up an appropriate model for the components $f_{Y_{j}}\left(y \mid y_{j-1}\right)$ seems to be more challenging. Therefore, we will focus only on this component here. Further information on the other components can be found in (Zimmermann, 2010). 
Although similarities can be expected for similar products to some extent, specific behavior may of course occur for specific data. Therefore, the following analysis has no ambition to be valid in general. The purpose of this chapter is to:

1. outline an appropriate method (which, however, may not be the only option for the given task); and to

2. illustrate the method on a specific portfolio with real properties and interpret the results to uncover properties that might be searched for in other practical portfolios.

The data used have real properties (real data with some noise). In order to preserve confidentiality, actual values of the parameters are mostly not published. Instead, a graphical representation of the results is presented. We believe that the specific values are not really important. Instead, we focus on properties or techniques that could be transferable to other portfolios. On purpose, we selected a group of claims that appears to be one of the most complex to model - the liability bodily claims. The maximum possible number of nonzero adjustments for each claim was empirically set to $m=11$. There were a few claims with more than eleven observed stages but eleven is believed to be 'sufficiently large' to cover the absolute majority of claims. All adjustments in stages higher than eleven were therefore treated as the adjustments in the eleventh stage. Annuities were excluded from this analysis since annuities require an entirely different model based on life insurance techniques.

Since the generalized linear models (GLM) are now commonly used for analogous purposes in the case of aggregate models, one was used as the modeling technique in the case of our model as well. As suggested in Anderson et al., 2007, and used, e.g., in Larsen, 2007, only factors (no covariates) were used. That means, instead of using the values $y_{s-1}$ directly, a discretized variable $\bar{Y}$ is defined with $Q$ discrete values denoted as $q=1,2, \ldots, Q$ for each observed claim (claims index $i$ is dropped) and each stage $s-1$. The discretization is displayed in Table 1 .

The reasoning for this approach is described in Anderson et al., 2007:

'Although variates do not require any artificially imposed categorization, the main disadvantage is that the use of polynomials may smooth over interesting effects in the underlying experience. Often it is better to begin modeling all variables as narrowly defined categorical factors (ensuring sufficient data in each category) and if the categorical factor presents GLM parameter estimates which appear appropriate for modeling with a polynomial, then the polynomial in the variate may be used in place of the categorical factor.'

Based on a pre-study, two highly significant interactions were identified. Namely the expected incurred value for stage 3 with a previous incurred value higher than 13.3 thousand (i.e., $s=3$ and $q \geq 3$ ) and for stage 11 and a previous incurred value higher than 22 thousand (i.e., $s=11$ and $q \geq 4$ ). In an ideal case, these 'anomalies' should be confirmed from a practical point of view (e.g. by claim handlers). However, this was not our case, so no interpretation can be provided for these interactions. 


\section{Table 1}

The discretization of the variable $Y_{s-1}$ :

The values $q$ of the variable $\bar{Y}_{s-1}$, the mean observation in each category $y_{q}^{+}$.

\begin{tabular}{|c|c|c|}
\hline$q$ & $Y_{s-1}$ & $Y_{s-1}^{+}$ \\
\hline 1 & $2-8103$ & 4073 \\
\hline 2 & $8104-13360$ & 10338 \\
\hline 3 & 13361 - 22026 & 17963 \\
\hline 4 & 22027 - 36316 & 28480 \\
\hline 5 & 36317 - 59874 & 48227 \\
\hline 6 & 59875 - 98716 & 72956 \\
\hline 7 & 98717 - 162755 & 120894 \\
\hline 8 & 162756 - 268337 & 205520 \\
\hline 9 & 268338 - 442413 & 327293 \\
\hline 10 & $442414-729416$ & 550733 \\
\hline 11 & 729417 - 1202604 & 922739 \\
\hline 12 & 1202605 - 1982759 & 1507401 \\
\hline 13 & 1982760 - 3269017 & 2537520 \\
\hline 14 & 3269018 - 5389698 & 4203913 \\
\hline 15 & $5389699-8886111$ & 6735369 \\
\hline
\end{tabular}

The (unconditional) distribution of the incurred value in the first stage $Y_{1}=X_{1}$ involves only well-known statistical methods such as histogram or parametric distribution fitting. Therefore, we focus in the following text only on modeling the conditional ('transition') distributions starting from $s=2$. The estimates performed in this chapter were calculated based on 7744 observations. The logarithmic link function and the gamma error distribution were considered. Thus, for each claim $i$ we assume a model of the form:

$$
E\left(Y_{i, s}\right)=\mu_{i, s}
$$

where

$$
\mu_{i, s} \equiv \exp \left(\eta_{i, s}\right)
$$

and

$$
\eta_{i, s}=\alpha_{0}+\sum_{j=2}^{m} \alpha_{j} \bar{s}_{j, s}+\sum_{q=1}^{Q} \beta_{q} \bar{y}_{q, i, s-1}+\sum_{u=1}^{2} \gamma_{u} \bar{r}_{u, i, s}
$$

where $\eta_{i, s}$ is the linear predictor, $\alpha_{0}$ is the intercept, $\overline{\boldsymbol{S}}_{j}$ is the dummy variable indicating in which stage $s$ the observation was observed, i.e., $\bar{s}_{j, s}=1$ for $s=j$, $\bar{s}_{j}=0$ otherwise, $\bar{y}_{q, i, s}$ is the dummy variable indicating whether the $i$-th claim 
was in the stage $(s-1)$ in the $q$-th category of the incurred value, and the last term incorporates the impact of the two interactions mentioned above (i.e., $\bar{r}_{u, i, s}$ indicates occurrence of the $u$-th interaction). $\alpha_{j}, \beta_{q}$ and $\gamma_{u}$ are the parameters. Notice that some parameters (of the reference levels) are redundant and will be set to 0 .

From a practical point of view, there is one property which can not be captured by a simple GLM model. The common GLM specification of the variance of the response variable is:

$$
\operatorname{var}\left(Y_{i, s}\right)=\varphi V\left(\mu_{i, s}\right)=\varphi V\left(\exp \left(\eta_{i, s}\right)\right)
$$

where $\varphi$ is the so-called scale (or dispersion) parameter and $V\left(\mu_{i, s}\right)$ is the variance function, which is for example in the case of logarithmic link function and the gamma distributed error $V\left(\mu_{i, s}\right)=\mu_{i, s}^{2}$. This means that the estimated response variance depends on the factors (and/or covariates) only through the estimated mean of the response variable. The scale parameter is a constant. Therefore, this model can not capture cases where the impact of the factors on the mean is different than the impact on the variance. From a practical point of view, we can expect a strong impact of the factor $S$ on the variance, which might be rather different from the impact of the factor $S$ on the mean response. Namely, it is probably natural that, in later stages, when more and more information about the claim arrives to the claim handlers, their estimate (present incurred value) of the ultimate incurred value should be more and more precise - the adjustments should only represent some 'fine tuning' of the loss. This can be translated as: We should expect the variance in the response to be decreasing with the stage $S$. Since there is no reason to assume that the mean response $E\left(Y_{i, s}\right)$ should exhibit such behavior, we can deduce from equation (28) that to capture this property, the scale parameter should be dependent on the stage $S$. (Namely the scale parameter can be expected to be decreasing with the stage $S$ ). Similar behavior might be observed for $Y_{s-1}$, i.e., it might be observed that the proportionality of the variance to the expected response is, e.g., lower for larger claims than for attritional claims. This means that we also need a model of the scale parameter. However, the model of the mean and the model of the scale parameter must be interlinked. These two problems can not be separated. This task is called 'joint modeling of mean and dispersion'. The literature on this topic is relatively limited. Some notes can be found in the article by England and Verrall (2002. A few pages on this topic are also mentioned in the monograph by McCullagh and Nelder (1989), which we summarize in the following text.

The main principle of the joint modeling is that for mean response, we use the same specification as in (25) - (27). This time, however, the specification of the response variance allows a factor-dependent (namely stage and previous incurred value dependent) scale parameter:

$$
\operatorname{var}\left(Y_{i, s}\right)=\varphi_{i, s} V\left(\mu_{i, s}\right)
$$

It is assumed that

$$
E\left(d_{i, s}\right)=\varphi_{i, s}
$$


where $d_{i, s}$ is some dispersion statistic and

$$
\varphi_{i, s} \equiv g_{D}\left(\xi_{i, s}\right)
$$

where $g_{D}$ is the dispersion link function and

$$
\xi_{i, s}=\sum_{j=2}^{m} \delta_{j} \bar{S}_{j, s}+\sum_{q=1}^{Q} \theta_{q} \bar{y}_{q, i, s-1}
$$

is the dispersion linear predictor and $\delta_{j}$ and $\theta_{q}$ are the parameters.

There are several options to choose from for the dispersion statistic $d_{i, s}$. In this paper, the squared Pearson residual was chosen, i.e.,

$$
d_{i, s} \equiv \frac{\left(Y_{i, s}-\mu_{i, s}\right)^{2}}{V\left(\mu_{i, s}\right)}=\frac{\left(Y_{i, s}-\mu_{i, s}\right)^{2}}{\mu_{i, s}^{2}} .
$$

In this paper, we again work with the logarithmic link function, i.e., we assume the multiplicative effect of the stage and the previous incurred value on the scale parameter and we again assume the gamma distributed error term. The specification of dispersion variance is

$$
\operatorname{var}\left(d_{i, s}\right)=\tau V_{D}\left(\varphi_{i, s}\right)
$$

where $V_{D}\left(\varphi_{s}\right)$ is the dispersion variance function, which is again in the case of logarithmic link function and the gamma distributed error $V_{D}\left(\varphi_{i, s}\right)=\varphi_{i, s}^{2}$.

In McCullagh and Nelder (1989), the authors state: 'The two models are interlinked; that for the mean requires an estimate of $1 / \varphi_{i, s}$ to be used as the prior weight, while the dispersion model requires an estimate of $\mu_{i, s}$ in order to form the dispersion response variable $d_{i}$. The form of the interlinking suggests an obvious algorithm for fitting these models, whereby we alternate between fitting the model for the means for given weights $1 / \hat{\varphi}_{i, s}$, and fitting the model for the dispersion using the response variable $d_{i, s}=d_{i s}\left(Y_{i, s}, \hat{\mu}_{i, s}\right)$.'

It is stated in England and Verrall (2006) that: 'If there is evidence that the scale parameter is not constant, simultaneous or 'joint' modeling of the mean and variance can be performed. Using maximum likelihood methods, this is an iterative process, whereby initial parameter estimates are obtained using arbitrary initial values for the scale parameters. The scale parameters are then updated, and revised parameter estimates obtained. The process iterates until convergence, although after the first iteration, the changes are usually small.'

The resulting parameter estimates provides an interesting insight into the properties of the observed process. The estimates of the parameters of the model of the mean response, i.e. model (27), are displayed in Figure 1 and 2 and 3 respectively. Based on 
the confidence intervals, one can state that in such a specified model, all the parameter estimates are statistically significant.

\section{Figure 1}

Estimates of the parameters (vertical axis) which are assigned to the dummy variables $\bar{S}_{j}$ coding the stage $S$ (horizontal axis). Dotted lines represent the upper and lower $95 \%$ confidence intervals.

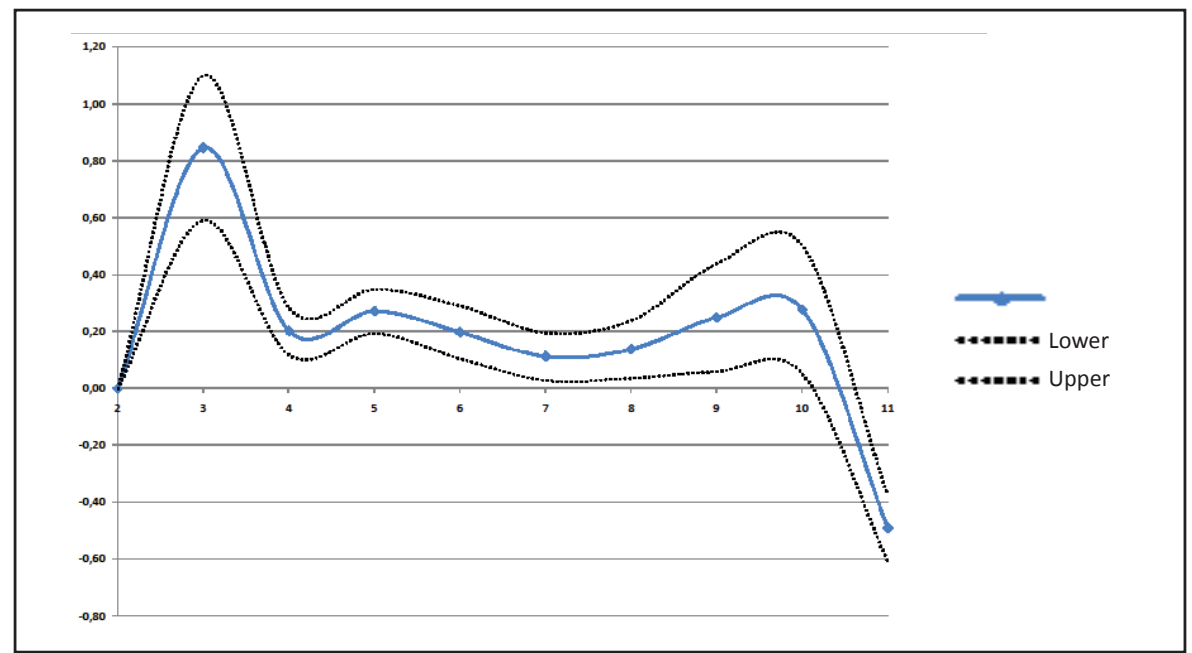

\section{Figure 2}

Estimates of the parameters (vertical axis) which are assigned to the dummy variables $\bar{y}_{q}$ coding the values of the discretized previous stage incurred value $\bar{Y}_{s-1}$. Dotted lines represent the upper and lower 95\% confidence intervals. On the horizontal axis, the category $q$ is represented by the mean of all the observations observed in the given category $y_{q}^{+}$.

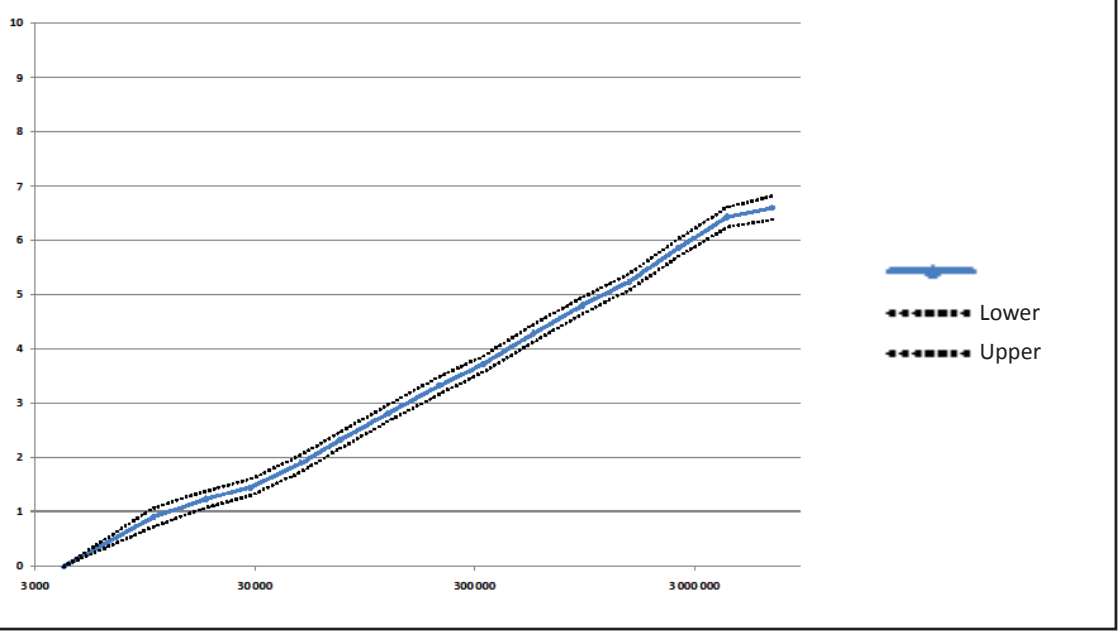


The estimates of the parameters assigned to the dummy variables representing the values of the stage (see Figure 1) suggest that in stage 3, one can generally expect a higher mean incurred value than in the reference stage (stage 2). This is also true for stages 4 to 10 , but the difference from the reference category is not so dramatic. In the last stage (stage 11), one can expect a lower mean incurred value than in the reference category. The estimates of the parameters assigned to the dummy variables representing the values of the previous incurred value $\bar{Y}_{s-1}$ (Fig. 2) are again all statistically significant. It seems that, if preferred, the original (undiscretized) values could be used as covariate with probably an additional effect of the smallest claims above the general trend. Both of the interaction parameter estimates were also statistically significant (see Figure 3).

\section{Figure 3}

Estimates of the parameters (vertical axis) which are assigned to the interaction dummy variables $r_{u}, u=1,2$ (horizontal axis) with the upper and lower 95\% confidence intervals.

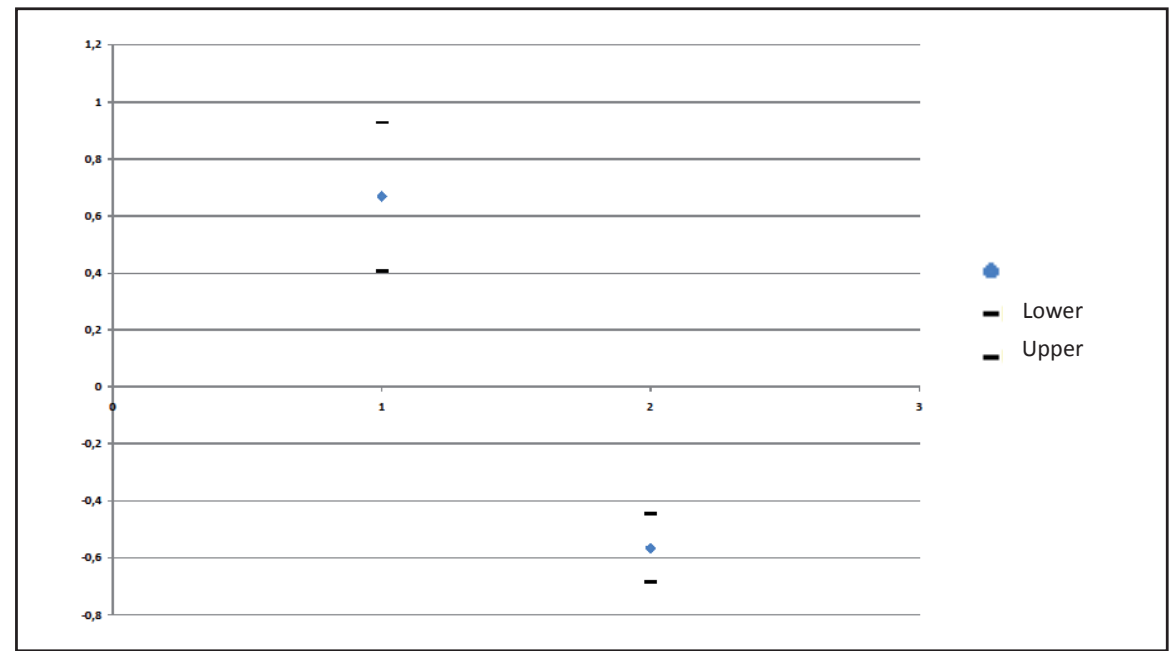

The estimates of the dispersion model parameters are displayed in Figures 4 and 5 respectively. All the parameter estimates were statistically significant. The parameter estimates assigned to the dummy variables representing the values of the stage suggest that the dispersion has a decreasing tendency with the stage, i.e., the results confirm the assumption that in general the claim handler's estimate becomes more and more precise during the settlement process. It is quite interesting that a similar result was also confirmed for the parameter estimates assigned to the dummy variables representing the discretized previous stage incurred values. The result can be interpreted as that the relative variability decreases with the size of the claim - if the claim is large, dramatic changes are less probable than for small claims. 
Figure 4

Model of the dispersion - Estimates of the parameters (vertical axis) which are assigned to the dummy variables $\bar{S}_{j}$ coding the stage $S$ (horizontal axis). Dotted lines represent the upper and lower $95 \%$ confidence intervals.

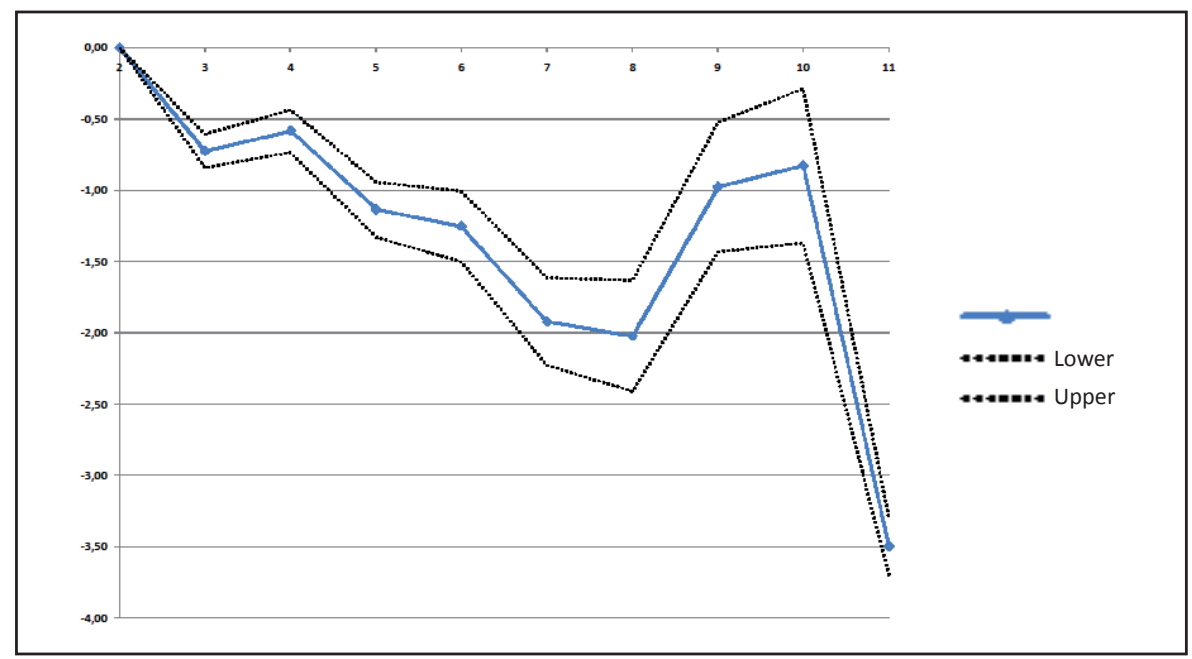

Figure 5

Model of the dispersion - Estimates of the parameters (vertical axis) which are assigned to the dummy variables $\bar{y}_{q}$ coding the values of the discretized previous stage incurred value $\bar{Y}$. Dotted lines represent the upper and lower $95 \%$ confidence intervals. On the horizontal axis, the category $q$ is represented by the mean of all observations observed in the given category $y_{q}^{+}$.

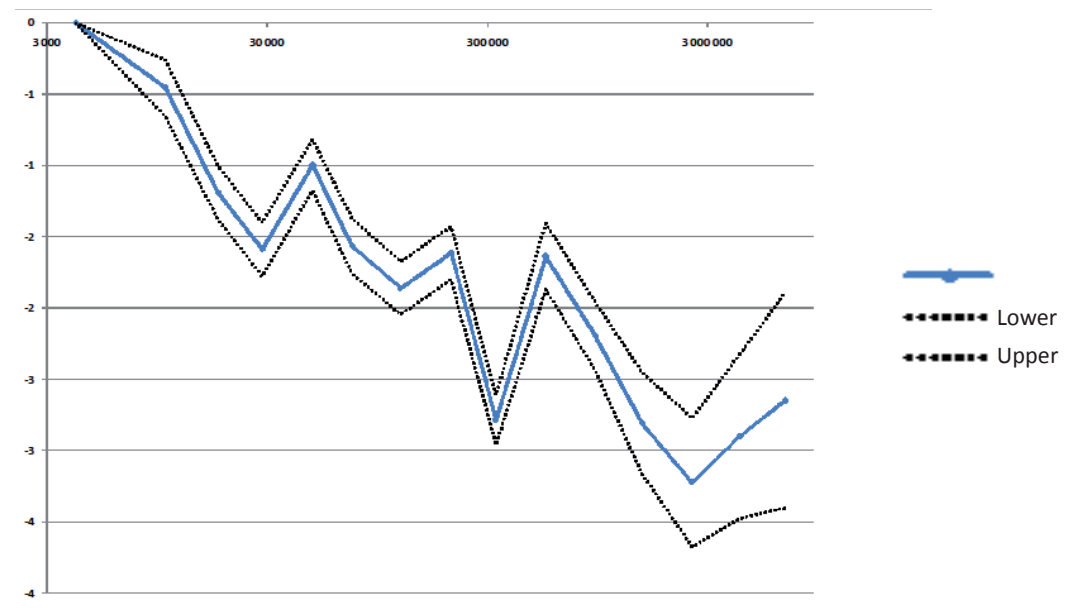




\section{Conclusions}

An analysis of possibilities of modeling the runoff of individual claims was studied in this article. The problem was decomposed into several sub-problems. This decomposition followed two goals:

1. It is necessary that the considered distributions can be modeled from the practical point of view, i.e. based only on data that can be expected to be available.

2. The model must be 'reasonably' complex in order to be applicable under real conditions.

One of the main focuses of this article was to point out simplifying assumptions that are not obviously wrong from a practical point of view on the one hand and that lead to a significant simplification of the model on the other hand. For the readers' convenience, we compile all the assumptions suggested in the above text here:

1. The ultimate incurred value of an individual claim is paid all at once at some 'average' time $T^{*}$.

2. A Markovian property of the series of the incurred value $Y_{1}, Y_{2}, \ldots, Y_{s}$ given that the claim is open.

3. The probability of a claim closure (given that the claim is open) only depends on the present incurred value (Markovian property of the series $A_{1}, A_{2}, \ldots, A_{s-1}$ ).

4. The settlement time $T^{*}$ only depends on the ultimate incurred value $Y$.

5. Knowledge of the maximum number of stages a claim can pass through (i.e., knowledge of $m$ ).

Of course, the assumptions made here can not be considered as always valid. On the other hand, the assumptions seem to be acceptable in a reasonable amount of practical cases and potential generalization of the assumptions outlined can be considered in many aspects.

\section{References}

ANDERSON, D.; FELDBLUM S.; MODLIN, C.; SCHIRMACHER, D; SCHIRMACHER, E.; THANDIC, N. 2007. A practitioners guide to generalized linear models. http://www.watsonwyatt.com/europe/ news/glmpaper/media/Practitioners_Guide.pdf.

ARJAS, E. 1989. The claims reserving problem in non-life insurance: Some structural ideas. ASTIN Bulletin, no. 19.

ENGLAND, P.D.; VERRALL, R.J. 2002. Stochastic claims reserving in general insurance. British Actuarial Journal. 2002 vol. 8, no. 3, p. 443-518.

ENGLAND, P.D.; VERRALL, R.J. 2006. Predictive distributions of outstanding liabilities in general insurance. Annals of Actuarial Science. 2006. vol. 1, no. 2, p. 221-270.

LARSEN, C.R. 2007. An individual claims reserving model. ASTIN Bulletin International Actuarial Association. 2007. vol. 37, no. 1, p.113-132. http://www.casact.org/library/astin/vol37no1/113.pdf.

MERZ M. and WUTRICH M. 2008. Modelling the claims development result for solvency purposes. Casualty Actuarial Society Forum Arlington, Virginia. 2008. p. 542-568. 
MACK, T. 1993. Distribution-free calculation of the standard error of chain-ladder reserve estimates. ASTIN Bulletin. 1993. vol. 23, p. 213-225.

MCCULLAGH, P.; NELDER, J.A. 1989. Generalized Linear Models. Second Edition. Chapman and Hall, 1989. ISBN: 0412317605

MURPHY, K.; McLennan, A. 2006. A method for projecting individual large claims. Casualty Actuarial Society Forum. 2006. p. 205 - 236. http://www.casact.org/pubs/forum/06fforum/209.pdf.

NORBERG, R. 1993. Prediction of outstanding liabilities in non-life insurance. ASTIN Bulletin International Actuarial Association.1993. vol. 23, no. 1, p. 95-115.

TAYLOR, G.; MCGUIRE G.; SULLIVAN, J. 2008. Individual claim loss reserving conditioned by case estimates. Annals of Actuarial Science. 2008. vol. 3, p. 215-256.

http://www.actuaries.org.uk/data/assets/pdffile/0016/24442/taylorreserving.pdf.

ZIMMERMANN, P. 2010. General Insurance Reserve Risk Modeling Based on Unaggregated Data. University of economics Prague, Czech Republic, 2010. PhD thesis.

\section{POSSIBILITIES OF INDIVIDUAL CLAIM RESERVE RISK MODELING}

Abstract: This article outlines possibilities of modeling the distribution of the future liabilities of an insurance company that stem from a past claim which has not yet been settled. Such a model might be used as a key component of the internal model of the reserve risk of an insurance company. It focuses on a probabilistic description of the settlement process of an individual loss, i.e., on the development of the incurred value over the lifetime of the loss. Such a model allows setting up an internal model based on an individual claim level instead of the aggregate claim level common nowadays. The proposed model respects two main restrictions given by potential industrial usage: Firstly, the model is set up in such a way that necessary data can be assumed to be available from a practical point of view. Besides that, potential requirements on the complexity of the model are considered and simplifying assumptions that allow setting up a model with 'reasonable complexity' for practical use are suggested and commented on from a practical point of view.

Calibration of the model of changes in the anticipated loss (more precisely, the incurred value changes) is illustrated on real data (adjusted for confidentiality purposes). The joint modeling procedure is applied, where a generalized linear model is assumed as a model of the incurred value (response variable) as well as its response variance. Interesting properties which might be expected in similar portfolios are revealed in the data.

Keywords: Claims reserving, Settlement process, Generalized Linear Model, Stochastic modeling

JEL Classification: C40, G03 